\title{
Ciclo de vida de Dysopeas muibum (Mollusca, Subulinidae) em laboratório: efeito do isolamento sobre padrões biológicos e conquiliomorfométricos
}

\author{
Vinícius J. Pilate ${ }^{1,2}$, Lidiane C. Silva ${ }^{2,3}$ \& Elisabeth C. A. Bessa ${ }^{2,4}$ \\ 1. Programa de Pós-graduação em Ciências Biológicas - Comportamento e Biologia Animal, Universidade Federal de Juiz de Fora, Juiz de Fora, MG, Brasil. (viniciuspilate@gmail.com) \\ 2. Núcleo de Malacologia da Universidade Federal de Juiz de Fora, Juiz de Fora, MG, Brasil. \\ 3. Programa de Pós-graduação em Ciências Veterinárias - Parasitologia Veterinária, Universidade Federal Rural do Rio de Janeiro, Seropédica, RJ, Brasil (lhybio@yahoo.com.br) \\ 4. Departamento de Zoologia, Instituto de Ciências Biológicas, Universidade Federal de Juiz de Fora, Juiz de Fora, MG, Brasil (elisabeth.bessa@ufjf.edu.br)
}

RESUMO. O conhecimento dos aspectos biológicos e morfológicos dos moluscos terrestres é importante para o desenvolvimento de medidas de controle de pragas e de estratégias de conservação de espécies. O presente trabalho teve como objetivo caracterizar os padrões de biologia e conquiliomorfometria de Dysopeas muibum Marcus \& Marcus, 1968 e verificar o efeito do isolamento sobre seu ciclo de vida e a morfometria da concha, em condições de laboratório. Foi realizado o acompanhamento do crescimento da concha, da liberação de filhotes e da mortalidade de 80 moluscos, dos quais 40 foram mantidos isolados e 40 agrupados (10 moluscos por grupo) em condições naturais de temperatura, umidade relativa e fotoperíodo, desde o nascimento até 180 dias de vida, quando então foram aferidas as medidas da concha. Os moluscos foram criados em terrários plásticos vedados com tecido de algodão e elástico, tendo como substrato terra vegetal esterilizada e alimentados com ração para frangos enriquecida com carbonato de cálcio. Foi verificado que os indivíduos possuem a concha pequena, alongada e são capazes de se reproduzir por autofecundação. Possuem crescimento indeterminado, iteroparidade, baixa mortalidade antes e após a maturidade sexual e a fecundidade aumenta de acordo com o tamanho corporal. $\mathrm{O}$ isolamento atuou na fecundidade, embora não tenha influenciado o crescimento, $\mathrm{o}$ alcance da maturidade sexual, a relação crescimento-reprodução, a mortalidade e a conquiliomorfometria.

PALAVRAS-CHAVE. Reprodução, Gastropoda, morfometria, concha.

\begin{abstract}
Life cycle of Dysopeas muibum (Mollusca, Subulinidae) in laboratory: effect of isolation on biological and conquiliomorphometric patterns. The knowledge of biological and morphological characteristics of terrestrial molluscs is important for the development of pest control measures and strategies for the conservation of species. This study aimed to characterize the patterns of biology and conquiliomorphometrics of Dysopeas muibum Marcus \& Marcus, 1968 and check the effect of isolation on its life cycle and morphometric of the shell, under laboratory conditions. We conducted monitoring growth of the shell, release of puppies and mortality of 80 molluscs, of which 40 were kept isolated and 40 grouped (10 molluses per group) in natural conditions of temperature, relative humidity and photoperiod, from birth to 180 days of life, when the measures of the shell were taken. The molluscs were reared in plastic terrariums sealed with cotton fabric and elastic, containing plant soil sterile, and fed with food to broilers enriched with calcium carbonate. It was found that the species has small and elongated shell, and is able to reproduce by self-fertilization. It has indeterminate growth, iteroparity, low mortality before and after sexual maturity and fecundity increases with body size. The isolation acted on fertility, although it has not influenced the growth, reach sexual maturity, growthreproduction relation, mortality and conquiliomorfometrics.
\end{abstract}

KEYWORDS. Reproduction, Gastropoda, morphometrics, shell.

O crescimento e a reprodução são aspectos importantes da biologia dos moluscos e estão intimamente relacionados, visto que a produção de ovos está ligada ao tamanho da concha (SIlva et al., 2008). As relações existentes entre os padrões de crescimento, reprodução e mortalidade, quando combinadas, representam os ciclos de vida das diferentes espécies de moluscos. O conhecimento dessas relações torna-se especialmente importante no contexto da conservação da biodiversidade, visto ser essencial para a estruturação de formas de manejo das populações de moluscos em risco de extinção (BACKELJAU et al., 2001).

Informações sobre o ciclo de vida também são necessárias para estabelecer estratégias de controle de parasitos que utilizam moluscos como hospedeiros intermediários (BofFI, 1979; D’ÁvILA et al., 2004), assim como de moluscos que atuam como pragas agrícolas (BoffI, 1979). O ciclo de vida e a morfologia dos moluscos terrestres podem ser influenciados por alterações nos fatores bióticos, como densidade populacional e competição (BAILEY, 1989; ANDERSON et al., 2007).

Dysopeas muibum Marcus \& Marcus, 1968 (Subulinidae) é encontrada em habitats úmidos e protegidos do sol e é nativa do continente americano; no Brasil foi registrada para as cidades de São Paulo, no estado de São Paulo e Juiz de Fora, em Minas Gerais (Simone, 2006; Pilate et al., 2012).

O objetivo deste estudo é caracterizar os padrões de crescimento, reprodução, mortalidade e conquiliomorfometria de D. muibum, descrevendo seu ciclo de vida em condições de laboratório, assim como verificar o efeito do isolamento sobre esses padrões.

\section{MATERIAL E MÉTODOS}

Foram utilizados 80 indivíduos recém-nascidos de D. muibum obtidos a partir de matrizes coletadas em uma horta do bairro Parque Independência, município de Juiz de Fora, Minas Gerais. Destes, 40 foram mantidos isolados em terrários plásticos transparentes de $100 \mathrm{~mL}$ ( $5 \mathrm{~cm}$ de diâmetro e $5 \mathrm{~cm}$ de altura) e 40 agrupados (quatro grupos, 10 moluscos por grupo) em terrários plásticos transparentes de $1000 \mathrm{~mL}(14 \mathrm{~cm}$ de diâmetro e $9 \mathrm{~cm}$ de altura), desde o nascimento até 180 dias de vida, entre março e setembro de 2011, compondo assim dois grupos experimentais. 
Fechados com tecido de algodão e elástico e umedecidos a intervalos de dois dias com $10 \mathrm{~mL}$ de água de torneira, os terrários de $100 \mathrm{~mL}$ continham como substrato $40 \mathrm{~g}$ ( $2 \mathrm{~cm}$ de altura) de terra vegetal esterilizada $\left(120^{\circ} \mathrm{C}\right.$ por uma hora) e os de $1000 \mathrm{~mL}, 200$ $\mathrm{g}$ (3 cm de altura). Os moluscos foram alimentados $a d$ libitum com ração para frangos peneirada e enriquecida com carbonato de cálcio (na proporção de 3:1), oferecida em discos plásticos transparentes de $4 \mathrm{~cm}$ de diâmetro (um disco por terrário) (BESSA \& ARAúJo, 1995a,b). Os terrários foram mantidos sob condições naturais e as temperaturas mínima e máxima e a umidade relativa do ar registradas diariamente com termômetro de máxima e de mínima e termo-higrômetro de bulbo seco e úmido, respectivamente. A média dos registros de temperatura foi de $20,30 \pm 2,24^{\circ} \mathrm{C}$ e de umidade relativa do ar, 78,90 $\pm 10,15 \%$

A intervalos quinzenais, a partir do primeiro dia do experimento até 180 dias de vida, foi medido com paquímetro o comprimento da concha dos dois grupos experimentais, para estabelecer o crescimento médio (mm) e a taxa de crescimento ( $\mathrm{mm}$ por dia) durante o ciclo de vida.

Para os indivíduos que atingiram 180 dias foram realizadas as seguintes medidas, adaptadas da metodologia usada por CHIU et al. (2002): comprimento da concha, largura da concha, comprimento da abertura, largura da abertura, comprimento da volta corporal, comprimento da penúltima volta e comprimento da espira; também foi determinado o número de voltas da concha. Com os valores destas variáveis lineares, foram calculadas razões, utilizadas como descritores da forma da concha: comprimento da concha/largura da concha, comprimento da volta corporal/comprimento da penúltima volta, comprimento da volta corporal/largura da concha, comprimento da abertura/largura da abertura e comprimento da espira/comprimento da volta corporal.

A observação de filhotes em terrários mantidos sob condição de isolamento foi o parâmetro utilizado para a constatação da ocorrência de autofecundação na espécie. $\mathrm{O}$ aparecimento de filhotes nos terrários, verificado através de observações diárias, foi o parâmetro utilizado para a constatação do tempo para o alcance da maturidade sexual pelos moluscos dos dois grupos experimentais. A partir desse dia até os 180 dias de vida, foram realizadas observações diárias para verificar o número de eventos reprodutivos realizados, o número de filhotes por evento reprodutivo e o intervalo entre eventos reprodutivos. Um evento reprodutivo foi caracterizado como um encontro de filhotes em um terrário, sendo todos quantificados, retirados e transferidos para as criações matrizes. Com esses dados, foi calculada a fecundidade (número médio de filhotes produzidos por evento reprodutivo e número médio de filhotes produzidos por molusco parental). No caso dos animais agrupados, o número de filhotes por indivíduo foi quantificado dividindo-se o número de filhotes em cada evento reprodutivo pelo número total de moluscos do grupo, por não ser possível saber quais moluscos do grupo se reproduziram e o número de filhotes que cada um procriou.

Para avaliar a taxa de mortalidade total, antes e após a maturidade sexual dos moluscos dos dois grupos experimentais, foram realizadas observações diárias desde o nascimento até 180 dias e cada morte observada foi registrada, sendo os animais mortos retirados do terrário.

Aplicou-se a análise de variância Kruskal-Wallis $(\mathrm{H})$, seguida pelo teste Student-Newman-Keuls (t) para a comparação das frequências médias dos parâmetros de crescimento, conquiliomorfometria, reprodução e mortalidade dos moluscos dos dois grupos experimentais. Para correlacionar o número médio de filhotes e o comprimento médio da concha ao longo dos 180 dias de experimento, foi utilizado o teste de Regressão Linear Simples (F). Na caracterização das relações de similaridade entre os padrões conquiliomorfométricos dos dois grupos experimentais, foi realizada a análise discriminante. Previamente, os valores das variáveis lineares das conchas foram submetidos à transformação logarítmica $\left(\log _{10}\right)$ para minimizar os desvios da normalidade nos valores brutos. As análises foram realizadas no programa BioEstat 5.0, adotando-se o índice de significância de 0,05 .

\section{RESULTADOS}

O comprimento da concha entre moluscos isolados e agrupados diferiu significativamente apenas na idade de 30 dias, sendo os valores maiores nos moluscos agrupados $(t=15,24 ; \mathrm{p}<0,01)$ (Fig. 1). Não houve diferença significativa no comprimento da concha entre moluscos isolados e agrupados no início do desenvolvimento $(\mathrm{H}=1,50 ; \mathrm{p}=0,22)$, no início da atividade reprodutiva $(\mathrm{H}=1,09 ; \mathrm{p}=0,30)$ e no final do experimento $(\mathrm{H}=0,32 ; \mathrm{p}=0,57)$ (Fig. 1$)$.

Observou-se que nos primeiros 30 dias de vida, os moluscos agrupados apresentaram um ritmo de crescimento mais rápido com relação aos isolados. Aos 45 dias, a situação se inverteu e, aos 60 , voltou à condição inicial. A partir dos 75 dias, a taxa de crescimento dos moluscos isolados e agrupados passou a ser semelhante no decorrer do tempo. Porém, tomando todo o período analisado, a taxa de crescimento não diferiu estatisticamente entre os dois grupos $(\mathrm{H}=0,05 ; \mathrm{p}=0,82)$. Dysopeas muibum apresentou padrão de crescimento indeterminado, com tendência à desaceleração, principalmente com a aproximação da maturidade sexual, uma vez que depois de maduros a taxa de crescimento diminui tanto em moluscos isolados $(t=4,46 ; p=0,04)$ quanto em agrupados $(t=4,43 ; p=0,02)$.

Na conquiliomorfometria, com relação às variáveis lineares, foi demonstrada diferença significativa entre moluscos isolados e agrupados apenas na largura da concha $(\mathrm{t}=8,76 ; \mathrm{p}=0,04)$ e no comprimento da penúltima 


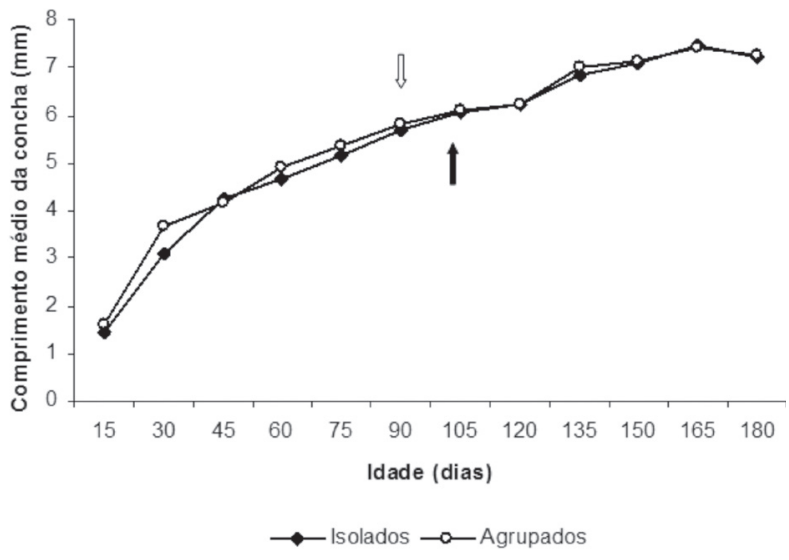

Fig. 1. Crescimento médio de Dysopeas muibum Marcus \& Marcus, 1968 mantidos sob condições de isolamento e agrupamento durante 180 dias de vida (setas indicam idades de alcance da maturidade sexual: seta preta - isolados; seta branca - agrupados).

volta $(\mathrm{t}=9,76 ; \mathrm{p}=0,03)$, sendo que as conchas dos indivíduos agrupados apresentaram os maiores valores dessas variáveis. Com relação às razões entre essas variáveis, não foi demonstrada diferença significativa em nenhuma delas (Tab. I).

Com base nas variáveis lineares da concha e nas razões entre essas variáveis, não foi possível a distinção de morfotipos entre moluscos isolados e agrupados, o que pode ser confirmado por meio da análise discriminante aplicada a essas variáveis (Fig. 2). Os padrões conquiliomorfométricos dos dois grupos foram muito próximos, não mostrando grande variação.

Em relação à reprodução, no decorrer de 180 dias de observação, constatou-se que indivíduos de D. muibum são capazes de se reproduzir por autofecundação, uma vez que 30 moluscos mantidos isolados desde o nascimento (75\%) foram capazes de gerar filhotes. Dos dez indivíduos que não produziram filhotes, oito morreram logo no início do experimento, sem atingir a maturidade sexual.

O tempo médio para alcançar a maturidade sexual nos indivíduos isolados foi de 101,23 $\pm 21,63$ dias (média dos indivíduos). Para os moluscos agrupados, esse período não foi significativamente diferente, 89 $\pm 6,93$ dias (média dos grupos), o que evidencia que o isolamento não causou alteração significativa do tempo para início da liberação de filhotes $(\mathrm{H}=1,41 ; \mathrm{p}=0,23)$.

Os moluscos isolados realizaram $9,17 \pm 2,65$ eventos reprodutivos, revertendo em 496 filhotes, enquanto os moluscos agrupados efetuaram 16,25 $\pm 2,87$ eventos reprodutivos, obtendo 295 filhotes. Constatouse que houve diferença significativa entre o número de eventos reprodutivos realizados por moluscos dos dois tratamentos $(\mathrm{t}=17,00 ; \mathrm{p}<0,01)$, assim como entre o número total de filhotes produzidos $(\mathrm{t}=17,00 ; \mathrm{p}<0,01)$. O intervalo entre eventos reprodutivos foi significativamente maior para os moluscos isolados $(8,64 \pm 7,20$ dias $)$ em relação aos agrupados $(5,85 \pm 3,45$ dias $)(t=13,03 ; \mathrm{p}=0,01)$.

Os moluscos isolados obtiveram 1,83 $\pm 0,43$ filhotes por evento reprodutivo e $16,53 \pm 5,16$ por molusco parental, enquanto os agrupados obtiveram $4,60 \pm 1,26$ filhotes por evento e 7,38 $\pm 2,08$ filhotes por molusco. A fecundidade observada em ambos os tratamentos diferiu, sendo reduzida pelo isolamento quanto ao número de filhotes por evento reprodutivo $(\mathrm{t}=17,00 ; \mathrm{p}<0,01)$, e maior quanto ao número de filhotes por molusco parental $(\mathrm{t}=16,15 ; \mathrm{p}<0,01)$.

O teste de Regressão Linear Simples mostrou ser significativa a relação entre crescimento e reprodução, representados respectivamente pelo comprimento médio da concha e pelo número médio de filhotes, tanto em moluscos isolados $\left(\mathrm{F}=17,71 ; \mathrm{p}<0,01 ; \mathrm{R}^{2}=0,60\right)($ Fig. 3$)$ quanto agrupados $\left(\mathrm{F}=10,74 ; \mathrm{p}=0,01 ; \mathrm{R}^{2}=0,47\right)$ (Fig. 4).

Tab. I. Média, desvio padrão, amplitude (valores mínimo e máximo) e coeficiente de variação das variáveis lineares da concha (mm) de Dysopeas muibum Marcus \& Marcus, 1968 mantidos sob condições de isolamento e agrupamento, com idade de 180 dias, e razões entre essas variáveis Letras diferentes indicam diferença significativa entre os grupos experimentais para a variável linear/razão analisada $(\mathrm{p}<0,05)(\mathrm{CV}$, coeficiente de variação; CA, comprimento da abertura; $\mathrm{CC}$, comprimento da concha; $\mathrm{CE}$, comprimento da espira; CPV, comprimento da penúltima volta; CVC, comprimento da volta corporal; DP, desvio padrão; LA, largura da abertura; LC, largura da concha; NV, número de voltas; CA/LA, razão entre comprimento da abertura e largura da abertura; CC/LC, razão entre comprimento da concha e largura da concha; CE/CVC, razão entre comprimento da espira e comprimento da volta corporal; CVC/CPV, razão entre comprimento da volta corporal e comprimento da penúltima volta; CVC/ LC, razão entre comprimento da volta corporal e largura da concha).

\begin{tabular}{|c|c|c|c|c|c|c|}
\hline & \multicolumn{3}{|c|}{ Isolados } & \multicolumn{3}{|c|}{ Agrupados } \\
\hline & Média \pm DP & Amplitude & CV (\%) & Média \pm DP & Amplitude & CV $(\%)$ \\
\hline $\mathrm{CC}$ & $7,20 \pm 0,59^{\mathrm{a}}$ & $(6,20-8,70)$ & 8,14 & $7,26 \pm 0,59^{\mathrm{a}}$ & $(6,00-8,40)$ & 8,08 \\
\hline $\mathrm{LC}$ & $2,30 \pm 0,26^{\mathrm{a}}$ & $(1,90-3,00)$ & 11,26 & $2,42 \pm 0,21^{b}$ & $(2,10-3,00)$ & 8,86 \\
\hline CA & $1,58 \pm 0,22^{\mathrm{a}}$ & $(1,20-2,00)$ & 13,92 & $1,63 \pm 0,21^{\mathrm{a}}$ & $(1,20-2,00)$ & 13,02 \\
\hline LA & $1,23 \pm 0,18^{a}$ & $(0,70-1,70)$ & 14,41 & $1,24 \pm 0,17^{\mathrm{a}}$ & $(0,90-1,60)$ & 13,34 \\
\hline $\mathrm{CVC}$ & $3,28 \pm 0,30^{\mathrm{a}}$ & $(2,70-3,90)$ & 9,18 & $3,34 \pm 0,28^{\mathrm{a}}$ & $(2,70-4,00)$ & 8,37 \\
\hline CPV & $1,47 \pm 0,23^{\mathrm{a}}$ & $(1,20-2,00)$ & 15,70 & $1,56 \pm 0,19^{b}$ & $(1,00-2,00)$ & 12,43 \\
\hline $\mathrm{CE}$ & $4,01 \pm 0,48^{\mathrm{a}}$ & $(3,10-5,40)$ & 11,97 & $3,93 \pm 0,57^{\mathrm{a}}$ & $(3,00-5,20)$ & 14,51 \\
\hline NV & $6,19 \pm 0,34^{a}$ & $(5,50-7,25)$ & 5,52 & $6,10 \pm 0,40^{\mathrm{a}}$ & $(5,25-7,00)$ & 6,62 \\
\hline $\mathrm{CC} / \mathrm{LC}$ & $3,15 \pm 0,32^{\mathrm{a}}$ & $(2,50-3,81)$ & 10,22 & $3,02 \pm 0,30^{\mathrm{a}}$ & $(2,46-3,62)$ & 10,03 \\
\hline $\mathrm{CVC} / \mathrm{CPV}$ & $2,27 \pm 0,37^{\mathrm{a}}$ & $(1,60-2,92)$ & 16,41 & $2,17 \pm 0,32^{\mathrm{a}}$ & $(1,69-3,20)$ & 14,60 \\
\hline CVC/LC & $1,44 \pm 0,20^{\mathrm{a}}$ & $(0,97-1,90)$ & 14,12 & $1,39 \pm 0,15^{\mathrm{a}}$ & $(1,04-1,62)$ & 10,85 \\
\hline CA/LA & $1,31 \pm 0,24^{\mathrm{a}}$ & $(1,00-2,14)$ & 18,50 & $1,33 \pm 0,21^{\mathrm{a}}$ & $(1,00-1,89)$ & 15,72 \\
\hline $\mathrm{CE} / \mathrm{CVC}$ & $1,23 \pm 0,20^{\mathrm{a}}$ & $(0,94-1,80)$ & 16,11 & $1,18 \pm 0,17^{\mathrm{a}}$ & $(0,75-1,49)$ & 14,04 \\
\hline
\end{tabular}




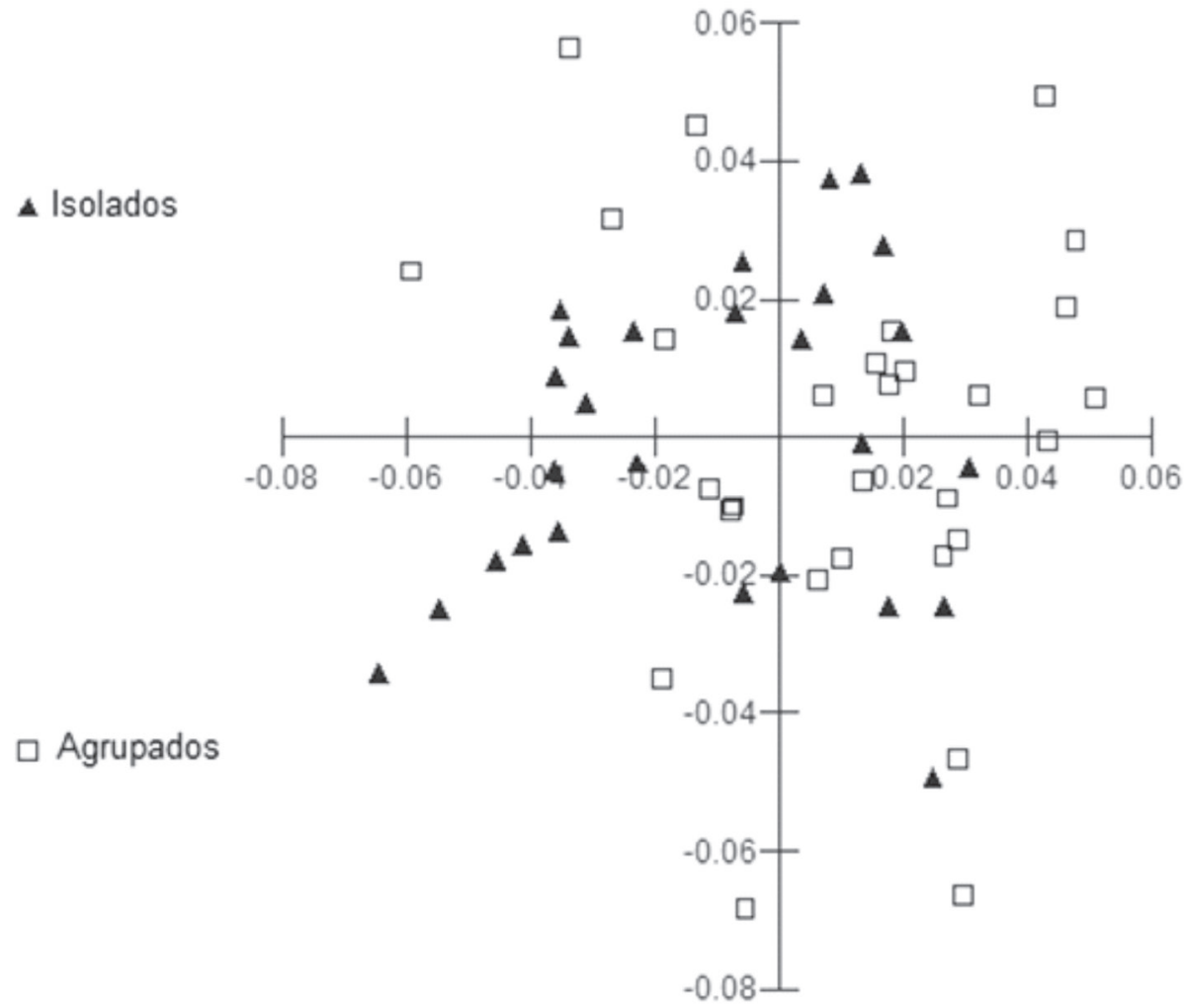

Fig. 2. Análise discriminante aplicada às variáveis lineares da concha de Dysopeas muibum Marcus \& Marcus, 1968 mantidos sob condições de isolamento e agrupamento, com idade de 180 dias de vida.

Com relação à mortalidade, no decorrer do experimento morreram 13 moluscos isolados (taxas de mortalidade pré-maturidade sexual de $23 \%$ e pósmaturidade sexual de $10 \%$ ) e 11 moluscos agrupados (taxas de mortalidade pré-maturidade sexual de $15 \%$ e pós-maturidade sexual de 13\%).

Não houve diferença significativa entre o número

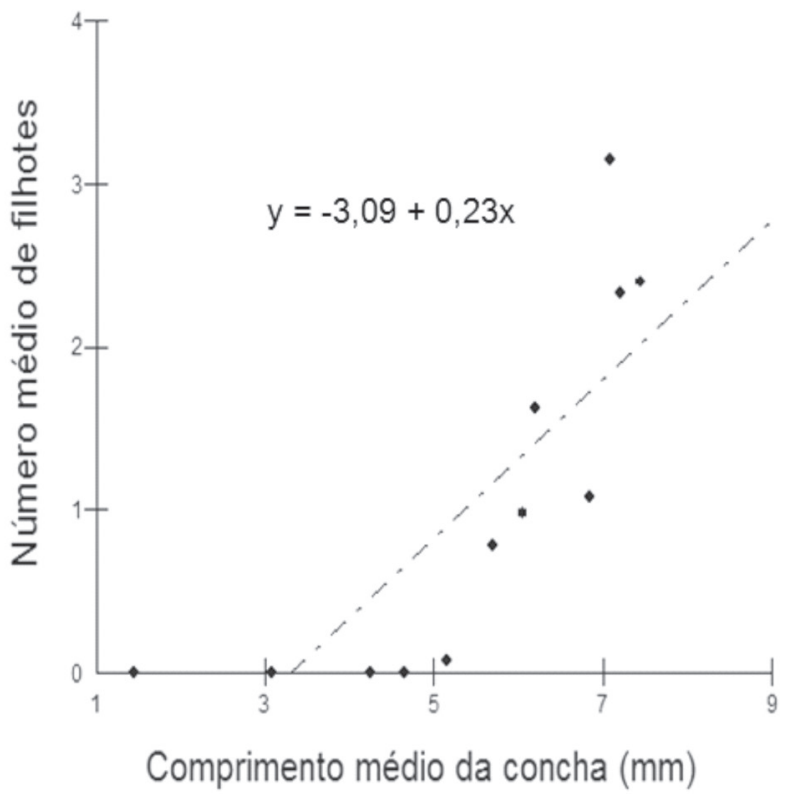

Fig. 3. Relação entre número médio de filhotes e comprimento médio concha de Dysopeas muibum Marcus \& Marcus, 1968 mantidos em condição de isolamento durante 180 dias de vida. total de moluscos mortos nos dois tratamentos durante todo o período estudado $(\mathrm{H}=0,01 ; \mathrm{p}=0,93)$, antes da maturidade sexual $(\mathrm{H}=0,04 ; \mathrm{p}=0,85)$ e depois da maturidade sexual $(\mathrm{H}=0,01 ; \mathrm{p}=0,93)$.

\section{DISCUSSÃO}

O comprimento médio da concha foi semelhante entre moluscos isolados e agrupados ao longo do estudo, assim como a taxa de crescimento. Como o alimento foi oferecido ad libitum e os moluscos agrupados estavam em um recipiente relativamente grande, levando-se em conta o tamanho médio dos exemplares, provavelmente não houve competição por recursos alimentares e espaço suficientemente para que fossem observadas modificações no crescimento e na conquiliomorfometria desta espécie.

Da mesma forma, o isolamento não influenciou o padrão de crescimento, pois nos dois tratamentos observou-se que no período anterior ao alcance da maturidade sexual a taxa de crescimento manteve-se alta e, após tal evento, tendeu a cair e estabilizar, comprovando que a espécie possui crescimento indeterminado, com tendência à desaceleração, principalmente com a aproximação do primeiro evento reprodutivo. Tal fato se deve aos moluscos investirem sua energia no crescimento antes de alcançarem a maturidade sexual e, ao alcançarem, investirem a maior parte de sua energia na reprodução, diminuindo assim o crescimento (HELLER, 


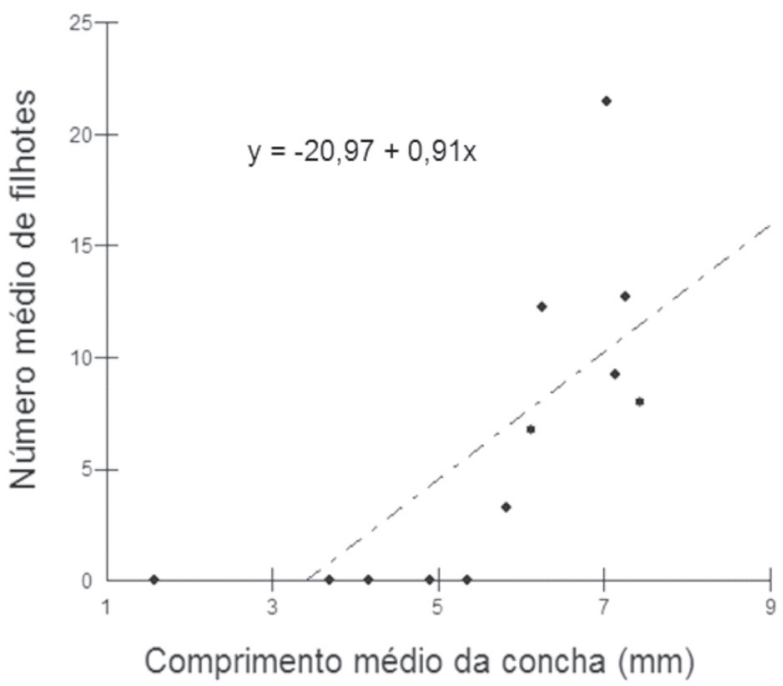

Fig. 4. Relação entre número médio de filhotes e comprimento médio da concha de Dysopeas muibum Marcus \& Marcus, 1968 mantidos em condição de agrupamento durante 180 dias de vida.

2001). Esse padrão também é observado em outros subulinídeos, como Subulina octona (Brugüière, 1792) (Bessa \& ARAúso, 1995a) e Leptinaria unilamellata (d'Orbigny, 1835) (Almeida \& Bessa, 2001a; CARvalHo et al., 2009), e em espécies de outras famílias de pulmonados terrestres como Bradybaena similaris (Férussac, 1821) (Xanthonychidae) (Almeida \& Bessa, 2001b; CARVAlHo et al., 2008), Bulimulus tenuissimus (d'Orbigny, 1835) (Bulimulidae) (SiLva et al., 2008) e Habroconus semenlini (Moricand, 1845) (Euconulidae) (SiLVA et al., 2009). No entanto, o crescimento após a maturidade não prejudica o sucesso reprodutivo, uma vez que a produção de ovos (ou filhotes) aumenta devido ao maior tamanho do corpo (Cichon, 1999; Heller, 2001).

Em D. muibum houve relação direta entre o comprimento médio da concha e o número médio de filhotes nos dois tratamentos, de modo que a fecundidade aumenta com o tamanho do corpo, como também foi verificado em Cepaea nemoralis (Linnaeus, 1758) (Helicidae) (Wolda \& Kreulen, 1973). Em S. octona, o crescimento do trato reprodutor está relacionado não só com o comprimento da concha, mas também com o peso do organismo parental (D’Ávila \& BesSA, 2005a,b,c).

As medidas lineares da concha de D. muibum, as razões entre essas medidas e a observação da morfologia externa permite caracterizar a concha como alongada e pequena. Estudos de variações nas medidas podem resultar na constatação de diferentes padrões morfométricos, permitindo inferir sobre características do animal, como forma do corpo e padrão de crescimento (ChIU et al., 2002). No presente trabalho, não se constatou variabilidade conquiliomorfométrica em indivíduos isolados e agrupados, uma vez que apenas houve diferença nas medidas da largura da concha e do comprimento da penúltima volta e em nenhuma razão entre medidas lineares. Assim sendo, não foi possível distinguir morfotipos diferentes para os dois tratamentos, com ambos apresentando forma alongada da concha, provavelmente pelos valores relativamente elevados das razões entre o comprimento e a largura da concha e entre o comprimento da espira e o comprimento da volta corporal.

Fatores bióticos como competição e densidade populacional podem influenciar a morfologia e a biologia de moluscos pulmonados terrestres (BAILEY, 1989; ANDERSON et al., 2007). Embora o isolamento um fator biótico - não tenha influenciado a morfologia desses animais, sabe-se que fatores abióticos como características do ambiente podem atuar na concha. Os pesquisadores observaram que moluscos de habitats úmidos e protegidos, como D. muibum, tendem a ter conchas com espiras altas e aberturas maiores (embora a abertura da concha de D. muibum seja pequena), enquanto os de locais secos e sem proteção, como áreas com alterações antrópicas, tendem a ter conchas com espiras baixas e aberturas estreitas (CHIBA \& Davison, 2007). Isso pode estar relacionado à menor retenção de umidade e temperaturas maiores nessas áreas, já que uma maior abertura leva a uma maior exposição do tegumento e, consequentemente, à desidratação. Além disso, o maior comprimento expõe o animal a predadores (SAntos \& Monteiro, 2001).

Os moluscos terrestres têm baixa capacidade de dispersão, de modo que a autofecundação é uma estratégia que possibilita um único indivíduo fundar uma nova população, diminuindo assim os riscos de extinção da espécie por falta de encontros com coespecíficos (GoMOT DE VAUfleurY, 2001). A autofecundação registrada nesse estudo é comum em moluscos dessa família, como verificado nos subulinídeos L. unilamellata (AlmeIDA \& BeSSA, 2001a) e S. octona (BESSA \& ARAúJo, 1995b) e em muitas outras famílias de gastrópodes terrestres (ARMBRUSTER et al., 2007). Essa estratégia foi verificada em muitos basomatóforos, como planorbídeos que se reproduzem apenas por esse modo, não havendo cópula, e não é incomum em estilomatóforos, apesar da maioria desses moluscos se reproduzir preferencialmente por fecundação cruzada (GoMot DE VAUfLeURY, 2001).

$\mathrm{O}$ isolamento não alterou o tempo para $\mathrm{o}$ alcance da maturidade sexual nessa espécie, dados que contrastam com os de outras como B. tenuissimus, onde moluscos mantidos em condições propícias à reprodução (agrupamento) iniciaram a produção e liberação de ovos em um tempo menor, o que consequentemente levou a um período jovem curto e a um tempo de vida adulta maior (SILVA et al., 2008).

Os animais mantidos agrupados apresentaram mais eventos reprodutivos e menor intervalo de tempo separando eventos consecutivos quando comparados aos isolados, como era esperado, uma vez que o agrupamento é uma condição propícia à reprodução. Porém, os moluscos isolados produziram mais filhotes até o término do experimento, no total e por molusco 
parental, embora tenham produzido menos filhotes por evento reprodutivo. Esses resultados mostram que, embora os moluscos agrupados tenham alcançado a maturidade mais cedo e tido mais eventos reprodutivos, tiveram menor fecundidade. Provavelmente houve ação dos mecanismos de seleção sexual, já que os moluscos agrupados devem ter realizado fecundação cruzada ao invés da autofecundação, onde o sucesso reprodutivo é maior, embora seja mais custosa em termos energéticos, uma vez que os animais precisam desempenhar o papel masculino e feminino, produzindo espermatozóides e oócitos (GómEz, 2001).

$\mathrm{O}$ isolamento não alterou o crescimento, embora tenha modificado a fecundidade, diferentemente do que ocorre em $B$. similaris que apresenta maior crescimento e menor fecundidade quando mantida em isolamento (Almeida \& Bessa, 2001b). O isolamento pode também, em espécies ovíparas, reduzir a eclodibilidade dos ovos, como registrado por Silva et al. (2008) em $B$. tenuissimus.

Neste estudo não foram observados ovos nos terrários, mas apenas filhotes, liberados diretamente do organismo parental. A retenção de ovos no organismo parental parece ser vantajosa durante condições ambientais desfavoráveis, como baixa umidade no ambiente, além de proteger da predação os filhotes, que vão sendo liberados apenas quando as condições voltam a ser favoráveis. Esta estratégia permite que os moluscos obtenham maior sucesso reprodutivo, por garantir a sobrevivência dos filhotes. Dentre os fatores que provocam a alteração na liberação da prole, destacamse as condições ambientais, a densidade populacional e a presença de predadores (HYMAN, 1967; BAUR, 1994; Heller, 2001).

A baixa mortalidade, tanto antes quanto após a maturidade sexual, é um componente importante do ciclo de vida dessa espécie, e é independente do modo de criação. Outras espécies da mesma família, tais como L. unilamellata e $S$. octona, também se caracterizam por baixa mortalidade depois do primeiro evento reprodutivo (Bessa \& AraúJo, 1995a; Almeida \& Bessa, 2001a; CARVAlHo et al., 2009).

O modo e a proporção como a energia é utilizada no ciclo de vida, principalmente na reprodução, leva à diferenciação de duas estratégias reprodutivas: a iteroparidade e a semelparidade. $\mathrm{O}$ investimento em mais de um ciclo reprodutivo, como verificado em $D$. muibum, denomina-se iteroparidade (STEARNS, 1989; HeLler, 2001).

$\mathrm{Na}$ iteroparidade verificada em $D$. muibum, os recursos energéticos foram distruibuídos durante o ciclo de vida, o que provavelmente favoreceu a fecundidade e a sobrevivência verificadas. A espécie se reproduz continuamente desde que alcança a maturidade sexual. As condições de laboratório como disponibilidade de alimento e umidade do substrato constantes podem ter favorecido a liberação de filhotes durante o período observado. Porém, vale ressaltar que este estudo não foi feito durante o ano todo e nem até o fim da vida de todos os indivíduos, não abordando assim aspectos de sazonalidade e a longevidade. Tais dados podem contribuir para maiores esclarecimentos sobre a estratégia de história de vida, como foi feito para $B$. similaris e L. unilamellata (CARVALHO et al., 2008, 2009).

Nos gastrópodes terrestres de clima tropical, o desenvolvimento do sistema reprodutor é geralmente contínuo (GómEZ, 2001). Entretanto, são necessários estudos de histologia desse sistema ao longo da longevidade para verificar se o processo de formação de gametas também é contínuo.

Agradecimentos. Agradecemos à Coordenação de Aperfeiçoamento de Pessoal de Nível Superior (CAPES) pelas bolsas concedidas.

\section{REFERÊNCIAS BIBLIOGRÁFICAS}

Almeida, M. N. \& Bessa, E. C. A. 2001a. Estudo do crescimento e da reprodução de Leptinaria unilamellata (d'Orbigny) (Mollusca, Subulinidae) em laboratório. Revista Brasileira de Zoologia 18(4):1107-1113.

2001b. Estudo do crescimento e da reprodução de Bradybaena similaris (Férussac) (Mollusca, Xanthonychidae) em laboratório. Revista Brasileira de Zoologia 18(4):1115-1122.

Anderson, T. K.; Weaver, K. F. \& Guralnick, R. P. 2007. Variation in adult shell morphology and life-history traits in the land snail Oreohelix cooperi in relation to biotic and abiotic factors. Journal of Molluscan Studies 73:129-137.

Armbruster, G. F. J.; Hofer, M. \& Baur, B. 2007. Effect of cliff connectivity on the genetic population structure of a rock-dwelling land snail species with frequent self-fertilization. Biochemical Systematics and Ecology 35:325-333.

Backeljau, T.; Baur, A. \& Baur, B. 2001. Population and conservation genetics. In: BARKER, G. M. ed. The biology of terrestrial molluscs. New York, CABI Publishing. 558p.

BAILEY, S. E. R. 1989. Foraging behavior of terrestrial gastropods: integrating field and laboratory studies. Journal of Molluscan Studies 55(2):263-272.

Baur, B. 1994. Parental care in terrestrial gastropods. Experientia 50:5-14.

Bessa, E. C. A. \& Araújo, J. L. B. 1995a. Oviposição, tamanho de ovos e medida do comprimento da concha em diferentes fases do desenvolvimento de Subulina octona (Brugüière) (Pulmonata, Subulinidae) em condições de laboratório. Revista Brasileira de Zoologia 12(3):647-654.

1995b. Ocorrência de autofecundação em Subulina octona (Brugüière) (Pulmonata, Subulinidae) em condições de laboratório. Revista Brasileira de Zoologia 12(3):719-723.

Boffi, A. V. 1979. Moluscos brasileiros de interesse médico e econômico. São Paulo, Hucitec. 182p.

Carvalho, C. M.; Bessa, E. C. A. \& D’Ávila, S. 2008. Life history strategy of Bradybaena similaris (Férussac, 1821) (Mollusca, Pulmonata, Bradybaenidae). Molluscan Research 28(3):171-174.

Carvalho, C. M.; Silva, J. P.; Mendonça, C. L. F.; Bessa, E. C. A. \& D’ÁviLA, S. 2009. Life history strategy of Leptinaria unilamellata (d'Orbigny, 1835) (Mollusca, Pulmonata, Subulinidae). Invertebrate Reproduction \& Development 53(4):211-222.

Chiba, S. \& Davison, A. 2007. Shell shape and habitat use in the north-west pacific land snail Mandarina polita from Hahajima, Ogasawara: current adaptation or ghost of species past? Biological Journal of the Linnean Society 91:149-159.

Chiu, Y. W.; Chen, H. C.; Lee, S. C. \& Chen, C. A. 2002. Morphometric analysis of shell and operculum variations in the viviparid snail, Cipangopaludina chinensis (Mollusca: Gastropoda), in Taiwan. Zoological Studies 41(3):321-331.

Cichon, M. 1999. Growth after maturity as a suboptimal strategy. Acta Oecologica 20(1):25-28 
D’Ávila, S. \& Bessa, E. C. A. 2005a. Influência do substrato sobre a reprodução de Subulina octona (Brugüière) (Mollusca, Subulinidae), sob condições de laboratório. Revista Brasileira de Zoologia 22(1):197-204.

.2005b. Influência do substrato sobre o crescimento de Subulina octona (Brugüière) (Mollusca, Subulinidae), sob condições de laboratório. Revista Brasileira de Zoologia 22(1):205-211.

2005c. Influência de diferentes substratos e umidade sobre o crescimento e número de ovos produzidos por Subulina octona (Brugüière) (Mollusca, Subulinidae), sob condições de laboratório. Revista Brasileira de Zoologia 22(2):349-353.

D’Ávila, S.; Dias, R. J. P.; Bessa, E. C. A. \& Daemon, E. 2004 Resistência à dessecação em três espécies de moluscos terrestres: aspectos adaptativos e significado para o controle de helmintos. Revista Brasileira de Zoociências 6(1):115-127.

Gómez, B. J. 2001. Structure and functioning of the reproductive system. In: BARKER, G. M. ed. The biology of terrestrial molluscs. New York, CABI Publishing. 558p.

Gomot DE Vaufleury, A. 2001. Regulation of growth and reproduction. In: BARKER, G. M. ed. The biology of terrestrial molluses. New York, CABI Publishing. 558p.

Heller, J. 2001. Life history strategies. In: BARKer, G. M. ed. The biology of terrestrial molluscs. New York, CABI Publishing. $558 \mathrm{p}$

Hyman, L. H. 1967. The invertebrates: Mollusca I. New York, McGraw-Hill. 792p.
Pilate, V. J.; Silva, L. C.; Vargas, T.; Souza, B. A.; Chicarino, E. D. \& BESSA, E. C. A. 2012. Repertório comportamental e horário de atividade do molusco terrestre Dysopeas muibum Marcus \& Marcus, 1978 (Mollusca, Subulinidae) em laboratório. BioFar Revista de Biologia e Farmácia 8(2):176-188.

Santos, S. B. \& Monteiro, D. P. 2001. Composição de gastrópodes terrestres em duas áreas do Centro de Estudos Ambientais e Desenvolvimento Sustentado (CEADS), Vila Dois Rios, Ilha Grande, Rio de Janeiro, Brasil - um estudo-piloto. Revista Brasileira de Zoologia 18(1):181-190.

Silva, L. C.; Meireles, L. M. O.; Junqueira, F. O. \& Bessa, E. C. A. 2008. Development and reproduction in Bulimulus tenuissimus (Mollusca: Bulimulidae) in laboratory. Revista Brasileira de Zoologia 25(2):220-223.

Silva, L. C.; Meireles, L. M. O.; Vargas, T.; Junqueira, F. O. \& Bessa, E. C. A. 2009. Life history of the land snail Habroconus semenlini (Stylommatophora: Euconulidae) under laboratory conditions. Revista de Biologia Tropical 57(4):1217-1222.

Simone, L. R. L. 2006. Land and freshwater molluses of Brazil. São Paulo, Fapesp. 390p.

Stearns, S. C. 1989. Trade-offs in life-history evolution. Functional Ecology 3(3):259-268

Wolda, H. \& Kreulen, D. A. 1973. Ecology of some experimental populations the landsnail Cepaea nemoralis (L.). II. Production and survival of eggs and juveniles. Netherlands Journal of Zoology 23(2):168-188. 